\title{
Avaliação da concentração de flúor em águas minerais comercializadas no Brasil*
}

\section{Evaluation of fluoride content of bottled drinking waters in Brazil}

\author{
Rita S. Villena, Denise G. Borges e Jaime A. Cury \\ Faculdade de Odontologia da Universidade de São Paulo. São Paulo, SP - Brasil (R.S.V., D.G.B.), \\ Laboratório de Bioquímica Oral da Faculdade de Odontologia da Universidade Estadual de \\ Campinas. Piracicaba, SP - Brasil (J.A.C.)
}

\begin{abstract}
Resumo
A utilização de flúor sistêmico deve ser controlada em termos de risco/benefício, pois enquanto a subdosagem não trouxer benefício anticárie, a sobredosagem estará associada com fluorose dental. Assim, uma das preocupações é o consumo de águas minerais, o qual tem aumentado nos últimos anos. Deste modo, foi realizado estudo com o objetivo de analisar a concentração de flúor em águas minerais comercializadas no Brasil. Foram analisadas 104 marcas comerciais, de diferentes regiões do País. Utilizou-se eletrodo específico Orion 96-09 e analisador de íons Orion EA 940, previamente calibrados com soluções padrões de flúor. Foram observadas concentrações de flúor variando de 0,0 a 4,4 ppm. Constataram-se: 1) Concentrações significativas de flúor não relatadas pelo produtor; 2) Concentrações de flúor sem significado preventivo anticárie, embora o produtor destaque na embalagem "Água Mineral Fluoretada"; 3) Concentrações de flúor capazes de provocar fluorose dental severa, embora o produtor não faça nenhuma referência que o mesmo existe. Conclui-se ser necessário um Sistema de Vigilância Sanitária para o controle de flúor nas águas minerais oferecidas à população brasileira, quer seja em termos de benefício como de risco.
\end{abstract}

Fluoração. Águas minerais, análise. Cárie dentária, prevenção \& controle.

\begin{abstract}
The utilization of a systemic fluoride method must be controlled in terms of risks and benefits. The reason for this is that while a small dose will not be effective in preventing dental caries, a larger dose may cause dental fluorosis. Therefore the knowledge of the fluoride concentration found in the bottled mineral waters sold throughout the Brazilian market the consumption of which has increased lately, is of great concern. The objective of the study was the analysis of the concentration of fluoride found in the bottled mineral waters. A hundred and four brands coming from different regions of Brazil were analysed using an Orion 96-09 ion specific electrode and an Orion EA 940 ionanalyser,
\end{abstract}

\footnotetext{
* Apresentado no $26^{\circ}$ Encontro do Grupo Brasileiro de Professores de Ortodontia e Odontopediatria, 1995 e ao $17^{\circ}$ Congresso Internacional de Odontologia de São Paulo, 1996.

Correspondência para/Correspondence to: Jaime A. Cury - Laboratório de Bioquímica Oral da Faculdade de Odontologia da Universidade Estadual de Campinas. Av. Limeira, 901 - 13414-018 Piracicaba, SP - Brasil Fax: (019) 421.0144 E-mail: fopdpt@turing.unicamp.br Edição subvencionada pela FAPESP. Processo 95/2290-6.

Recebido em 9.1.1996. Aprovado em 3.4.1996.
} 
previously calibrated with standard fluoride solutions. Different concentrations of fluoride ranging from 0.0 to 4.4 were found. It was discovered that specific bottled waters contained: 1) Significant concentrations of fluoride not reported by the producer; 2) Fluoride concentrations of no preventive effect, although the producer had advertised the water as a Fluoridated Mineral Water; 3) Fluoride concentrations high enough to cause dental fluorosis, although the producer did not alert the consumer to this fact. It is to be concluded, therefore, that a sanitary regulatory system for the control of the level of fluoride in the bottled mineral waters marketed is necessary. Such reputation should be formulated in terms of benefits as well as in terms of risks.

Fluoridation. Mineral waters, analysis. Dental caries, prevention and control.

\section{INTRODUÇÃO}

O Brasil é um País rico em água subterrânea, inclusive em regiões afetadas pela seca ${ }^{3}$. Dados de 1990 mostraram 202 concessões de lavras existentes para exploração de fontes de água mineral em todo o território, obtidas junto ao Ministério das Minas e Energia, órgão responsável pela regulamentação ${ }^{4}$.

Em 1993, a região Sudeste respondeu por 55,37\% da produção nacional de água mineral, a região Nordeste por $21,42 \%$, a região Sul por $11,84 \%$, a Centro-Oeste por $6,13 \%$ e a Norte por $5,24^{3}$. Ao mesmo tempo, São Paulo foi o Estado que mais engarrafou água mineral e potável de mesa $(37,44 \%)$, seguido por Minas Gerais $(9,14 \%)$, Pernambuco $(7,10 \%)$, Rio de Janeiro (6,83\%), Ceará (6,16\%), Paraná (4,24\%), Santa Catarina $(3,97 \%)$, Bahia $(3,95 \%)$ e Rio Grande do $\operatorname{Sul}(3,62 \%)^{3}$.

O consumo de água mineral engarrafada no Brasil apresenta um crescimento contínuo, principalmente nas regiões Nordeste e Sudeste ${ }^{3}$. Em 1993, o consumo nacional foi da ordem de 1.109 milhões de litros, o que representou um crescimento de $26 \%$ em relação a $1992^{3}$. Geralmente os consumidores de água mineral buscam uma água saudável, livre de impurezas para uso diário, em especial para crianças; ou seus efeitos medicinais benéficos para a saúde e digestão. Sua utilização vai desde o consumo "in natura", até o preparo de alimentos, gelo, sucos e bebidas $^{23,32}$.

As águas minerais engarrafadas deveriam seguir os mesmos critérios estabelecidos pela portaria 56 de 14/03/77, do Ministério da Saúde, publicada no
Diário Oficial da União do dia 15/06/77 que regulamenta o padrão de potabilidade da água no território nacional. Ela estabelece que o Valor Máximo Permissivel para o íon flúor na água potável, é de 0,6 a $1,7 \mathrm{mg} / \mathrm{l}^{*}$. Valores acima destes classificariam a água como não potável ${ }^{5}$. A Organização Mundial de Saúde (1984) estabelece que a concentração máxima de flúor na água potável em termos de fluorose dental seria de 1,5 ppm ${ }^{37}$. Já a Agência de Proteção Ambiental Americana estabeleceu, em 1986, o limite em 2 ppm, e com relação à fluorose óssea, em 4 ppm $^{34}$. Considerando os relatos na literatura mundial de fluorose óssea, causada pela ingestão crônica de águas engarrafadas com altos teores de flúor ${ }^{1,15,19,27}$, é também de grande importância que se mantenha controle da composição química das águas minerais nacionais. Por outro lado, pela falta de fiscalização, alguns fabricantes se valem do apelo promocional do flúor**, classificando como "fluoretadas" águas que não possuem concentrações que promoveriam um efeito cariostático preventivo. Isto em função do Código de Águas Minerais² da legislação brasileira permitir que se classifique como fluoretadas, aquelas que acusem em sua composição o íon.

Levy e col. ${ }^{23}$ entrevistaram um grupo de 107 mães, onde em média $18 \%$ dos bebês consumiam água mineral do nascimento aos 18 meses. Flaitz e col. ${ }^{13}$ pesquisaram o consumo diário de água mineral em 1.126 pacientes infantis de uma clínica privada no Colorado ${ }^{1}$, e encontraram uma prevalência de consumo equivalente a 9,3\%. Ao ser avaliada a concentração de flúor das águas minerais consumidas, observou-se que apenas $10 \%$ dessas crianças rece-

\footnotetext{
* mg/l: miligramos por litro

** Termo genérico no Brasil, entretanto no presente trabalho refere-se a ión flúor (fluoreto).
} 
biam a dosagem ideal de flúor, entretanto o fato preocupante é que $72,4 \%$ desses consumidores recebiam uma sobredosagem. Além disso, $10 \%$ dessas crianças também recebiam suplementação de flúor indicada por seus pediatras, embora ingerissem água mineral contendo entre 1,0 e 1,4 ppm de flúor.

Clovis e Hargreaves ${ }^{9}$ mostraram que mesmo em cidades sem fluoretação da água, a ingestão diária de flúor, através de bebidas, pode atingir níveis ótimos ou mesmo superiores, desde que estejam à disposição da população bebidas com flúor em sua composição, fabricadas em cidades com fluoretação na água de abastecimento.

A literatura internacional tem mostrado preocupação acerca deste assunto, que pode ser constatada

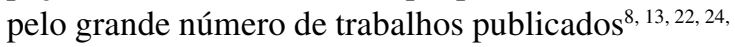
26,31, 36. No Brasil Franco e Maltz ${ }^{14}$, em 1991, estudaram a concentração de fluoretos em 8 águas minerais comercializadas no Rio Grande do Sul. Uma delas apresentou uma concentração elevada de flúor (2,94 ppm), duas apresentaram concentrações dentro dos níveis indicados $(0,75$ a 0,93 ppm) e a maioria, as seis amostras restantes continham baixos níveis $(0,1$ a $0,62 \mathrm{ppm})$. Por este motivo, no presente estudo decidiu-se coletar o maior número possível de amostras de águas minerais disponíveis no mercado nacional, para que os profissionais possam ter uma noção dos teores de flúor encontrados nos diferentes Estados brasileiros, considerando os aspectos de risco/benefício.

\section{MATERIAL E MÉTODO}

Foram analisadas 104 marcas comerciais de águas minerais adquiridas no mercado de vendas brasileiro, de agosto de 1994 até fevereiro de 1996. Do total da amostra, 96 foram águas da indústria nacional provenientes de 16 Estados e 8 foram de origem estrangeira, adquiridas no comércio da cidade de São Paulo.

\section{Determinação de Flúor nas Águas Minerais}

As amostras de água foram analisadas em duplicata, utilizando um analisador de íons, ORION EA 940, e um eletrodo específico, ORION 96-09, previamente calibrados com soluções padrões, contendo de 0,1 a 1,0 $\mu \mathrm{g} \mathrm{F} / \mathrm{ml}$, preparados em TISAB* a 50\%. Foi utilizado o método de leitura direta, após a colocação de $1 \mathrm{ml}$ da amostra de água e $1 \mathrm{ml}$ do TISAB, sendo o aparelho recalibrado após a leitura de 20 amostras. A precisão das análises foi determinada com padrão ORION (940907).

\section{RESULTADOS}

As concentrações de flúor das 104 águas mineriais avaliadas variaram de 0,0 a 4,4 ppm, e são apresentadas nas Tabelas 1 a 6 .

Com a finalidade de facilitar a interpretação dos resultados, as concentrações de flúor relatadas na forma de $\mathrm{NaF}$ ou KF pelos fabricantes foram apresentadas nas tabelas como flúor iônico (ppm F).

Tabela 1 - Concentrações de flúor (ppm) encontradas e relatadas em águas minerais comercializadas nos Estados de Amazonas, Bahia, Brasília, Ceará, Espírito Santo e Goiás.

\begin{tabular}{lccc}
\hline \multicolumn{1}{c}{$\begin{array}{c}\text { Procedência } \\
\text { (Estado) }\end{array}$} & $\begin{array}{c}\text { Código das águas } \\
\text { minerais }\end{array}$ & \multicolumn{2}{c}{ ppm F } \\
Relatado & Encontrado \\
\hline Amazonas & A-1 & N.E.* & 0,04 \\
Bahia & B-1 & N.E. & 0,00 \\
Brasília & B-2 & N.E. & 0,00 \\
Ceará & BR-1 & N.E. & 0,00 \\
& C-1 & N.E. & 0,01 \\
& ES-1 & N.E. & 0,49 \\
Espírito Santo & ES-2 & N.E. & 0,02 \\
& ES-3 & N.E. & 0,01 \\
& ES-4 & 0,04 & 0,00 \\
Goiás & ES-5 & N.E. & 0,00 \\
* Não especificado pelo fabricante & N.E. & 0,00 \\
& G-1 & 0,10 & 0,00 \\
\hline
\end{tabular}

Tabela 2 - Concentrações de flúor (ppm) encontradas e relatadas em águas minerais comercializadas nos Estados de Minas Gerais, Pará e Paraná.

\begin{tabular}{lccc}
\hline $\begin{array}{l}\text { Procedência } \\
\text { (Estado) }\end{array}$ & $\begin{array}{c}\text { Código das águas } \\
\text { minerais }\end{array}$ & \multicolumn{2}{c}{ ppm F } \\
\hline Mato Grosso & M-1 & N.E. & 0,04 \\
& MG-1 & N.E. & 0,24 \\
& MG-2 & 0,29 & 0,13 \\
Minas Gerais & MG-3 & N.E. & 0,12 \\
& MG-4 & 0,02 & 0,11 \\
& MG-5 & N.E. & 0,11 \\
& MG-6 & N.E. & 0,08 \\
& MG-7 & N.E. & 0,04 \\
& MG-8 & N.E. & 0,03 \\
& MG-9 & N.E. & 0,01 \\
& MG-10 & N.E. & 0,00 \\
& P-1 & N.E. & 0,00 \\
& P-2 & N.E. & 0,00 \\
& PR-1 & N.E. & 0,93 \\
& PR-2 & N.E. & 0,04 \\
& PR-3 & N.E. & 0,03 \\
Paraná & PR-4 & N.E. & 0,03 \\
& PR-5 & N.E. & 0,02 \\
& PR-6 & N.E. & 0,00 \\
\hline
\end{tabular}


Tabela 3 - Concentrações de flúor (ppm) encontradas e relatadas em águas minerais comercializadas nos Estados de Pernambuco, Piauí e Rio de Janeiro.

\begin{tabular}{lccc}
\hline $\begin{array}{l}\text { Procedência } \\
\text { (Estado) }\end{array}$ & $\begin{array}{c}\text { Código das águas } \\
\text { minerais }\end{array}$ & \multicolumn{2}{c}{ ppm F } \\
\hline Pernambuco & PE-1 & N.E. & 0,0 \\
Piauí & PE-2 & N.E. & 0,0 \\
& PI-1 & N.E. & 0,02 \\
& RJ-1 & N.E. & 0,28 \\
Rio de Janeiro & RJ-2 & 0,01 & 0,10 \\
& RJ-3 & 0,02 & 0,10 \\
& RJ-4 & 0,31 & 0,04 \\
& RJ-5 & N.E. & 0,00 \\
\hline
\end{tabular}

Tabela 4 - Concentrações de flúor (ppm) encontradas e relatadas em águas minerais comercializadas nos Estados de Rio Grande do Sul e Santa Catarina.

\begin{tabular}{lccc}
\hline $\begin{array}{l}\text { Procedência } \\
\text { (Estado) }\end{array}$ & $\begin{array}{c}\text { Código das águas } \\
\text { minerais }\end{array}$ & \multicolumn{2}{c}{ ppm F } \\
& RG-1 & 4,40 & 4,44 \\
& RG-2 & 1,83 & 1,69 \\
Rio Grande & RG-3 & 1,83 & 1,66 \\
do Sul & RG-4 & 0,9 & 0,87 \\
& RG-5 & N.E. & 0,73 \\
& RG-6 & N.E. & 0,52 \\
& RG-7 & 0,09 & 0,15 \\
& RG-8 & 0,02 & 0,14 \\
& SC-1 & N.E. & 1,02 \\
& SC-2 & N.E. & 0,68 \\
Santa Catarina & SC-3 & 0,36 & 0,34 \\
& SC-4 & 0,15 & 0,17 \\
& SC-5 & N.E. & 0,07 \\
& SC-6 & N.E. & 0,01 \\
\hline
\end{tabular}

Tabela 6 - Concentrações de flúor (ppm) encontradas e relatadas em águas minerais importadas comercializadas na cidade de São Paulo.

\begin{tabular}{lccc}
\hline $\begin{array}{l}\text { Procedência } \\
\text { (Estado) }\end{array}$ & $\begin{array}{c}\text { Código das águas } \\
\text { minerais }\end{array}$ & \multicolumn{2}{c}{$\mathrm{ppm} \mathrm{F}$} \\
\hline Pelatado & Encontrado \\
\hline Portugal & I-1 & 2,50 & 3,16 \\
Argentina & I-2 & 2,00 & 1,89 \\
Itália & I-3 & N.E. & 0,68 \\
Alemanha & I-4 & N.E. & 0,31 \\
Escócia & I-5 & N.E. & 0,17 \\
França & I-6 & N.E. & 0,11 \\
França & I-7 & 0,12 & 0,06 \\
Portugal & I-8 & $<0,05$ & 0,03 \\
\hline
\end{tabular}

As avaliações mostraram que $84(80,8 \%)$ das águas minerais apresentavam concentrações de flúor, que variavam de 0,0 a 0,29 ppm, $9(8,6 \%)$ apresentaram variações de 0,3 a 0,7 ppm e $11(10,6 \%)$ mostraram concentrações maiores de $0,7 \mathrm{ppm}$, sendo que $8(7,7 \%)$ delas continham mais de $1 \mathrm{ppm} \mathrm{F}$.

Ao verificar os rótulos das embalagens e compará-los aos resultados, comprovou-se que duas águas
Tabela 5 - Concentrações de flúor (ppm) encontradas e relatadas em águas minerais comercializadas no Estado de São Paulo.

\begin{tabular}{|c|c|c|}
\hline \multirow{2}{*}{$\begin{array}{c}\text { Código das águas } \\
\text { minerais }\end{array}$} & \multicolumn{2}{|c|}{ ppm F } \\
\hline & Relatado & Encontrado \\
\hline S-1 & 0,64 & 1,36 \\
\hline S-2 & 0,90 & 0,92 \\
\hline S-3 & N.E. & 0,63 \\
\hline S-4 & N.E. & 0,46 \\
\hline S-5 & 0,18 & 0,31 \\
\hline S-6 & N.E. & 0,27 \\
\hline S-7 & 0,42 & 0,24 \\
\hline S-8 & 0,07 & 0,16 \\
\hline S-9 & N.E. & 0,15 \\
\hline S-10 & N.E. & 0,14 \\
\hline S-11 & N.E. & 0,14 \\
\hline S-12 & N.E. & 0,13 \\
\hline S-13 & N.E. & 0,13 \\
\hline S-14 & 0,78 & 0,12 \\
\hline S-15 & 0,25 & 0,11 \\
\hline S-16 & N.E. & 0,09 \\
\hline S-17 & N.E. & 0,09 \\
\hline S-18 & 0,10 & 0,08 \\
\hline S-19 & N.E. & 0,07 \\
\hline S-20 & N.E. & 0,07 \\
\hline S-21 & N.E. & 0,07 \\
\hline$S-22$ & N.E. & 0,06 \\
\hline S-23 & N.E. & 0,06 \\
\hline S-24 & N.E. & 0,06 \\
\hline S-25 & N.E. & 0,05 \\
\hline S-26 & N.E. & 0,05 \\
\hline S-27 & N.E. & 0,04 \\
\hline S-28 & N.E. & 0,04 \\
\hline S-29 & N.E. & 0,04 \\
\hline S-30 & N.E. & 0,04 \\
\hline S-31 & N.E. & 0,03 \\
\hline S-32 & 0,06 & 0,03 \\
\hline S-33 & 0,24 & 0,03 \\
\hline S-34 & N.E. & 0,03 \\
\hline S-35 & N.E. & 0,02 \\
\hline S-36 & N.E. & 0,02 \\
\hline S-37 & 0,42 & 0,01 \\
\hline S-38 & N.E. & 0,01 \\
\hline S-39 & N.E. & 0,01 \\
\hline$S-40$ & N.E. & 0,00 \\
\hline S-41 & N.E. & 0,00 \\
\hline S-42 & N.E. & 0,00 \\
\hline S-43 & N.E. & 0,00 \\
\hline
\end{tabular}

são comercializadas como fluoretadas, mas não contêm flúor na sua composição (G-2 - GO e S-37 - S.P.), sete contêm flúor e não especificam (E-1 - E.S., PR-1 - P.R., S-3 - SP, RG-5 - R.S., RG-6 - RS, SC-1 - S.C., SC-2 - S.C., S-4 - S.P., I-4 - Alemanha e I-3 - Itália). Outras cinco indicam ter uma concentração de flúor consideravelmente maior do que na verdade contêm (MG-3 - M.G., SC-3 - S.C., RJ-4 - R.J, S-14 - SP e S- 
2 - S.P.), e finalmente três outras são comercializadas como "águas fluoretadas", embora sua concentração seja mínima, não proporcionando efeitos cariostáticos (RJ-2 - R.J., S-32 - S.P. e SC-4 - S.C.).

Mediante esta avaliação verificou-se que 10 $(9,6 \%)$ das 104 marcas comerciais não apresentavam o flúor dentro de seus componentes, sendo que através das determinações, estas águas minerais continham mais de 0,3 ppm F.

\section{DISCUSSÃO}

Um incremento no consumo de água mineral, vem sendo constatado no Brasil ${ }^{3}$ e em outros países ${ }^{13,26 \text {, }}$ ${ }^{33,36}$, talvez devido à procura de uma água saúdavel, livre de impurezas e destinada ao consumo diário, em especial para as crianças ${ }^{36}$.

Geralmente presume-se que crianças até os dois anos de idade, que são amamentadas natural e/ou artificialmente e que consomem exclusivamente água mineral, não recebam flúor sistêmico. Acredita-se que sua ingestão é menor que $0,3 \mathrm{mg}$ de flúor/dia. Devido a este tipo de colocação, alguns profissionais indicam como medida alternativa a suplementação de flúor. Schalka e Rodrigues ${ }^{29}$ através de questionários, verificaram que a freqüência de prescrições de flúor sistêmico entre os pediatras de São José dos Campos foi de $18 \%$. Já Soviero e col..$^{30}$ em Petrópolis, acharam que $71,8 \%$ dos pediatras prescreviam suplementos fluoretados, sendo que $75,9 \%$ dos profissionais classificaram-no como um procedimento de rotina. Porém, só $41,4 \%$ deles responderam que antes de prescrever era necessário o conhecimento prévio do flúor na água de abastecimento. Dados similares também foram encontrados na literatura americana, variando de 49 a $97 \%$ na sua freqüência de prescriçãao ${ }^{10,16,17,21,25}$. Este tipo de resultado sugere que há necessidade de uma revisão e atualização de conceitos $^{18,23}$, com a finalidade de não submeter os pacientes infantis ao risco de sobredosagens.

Um fator a ser considerado é a dose máxima de flúor que deve ser ingerida diariamente, e que segundo Burt ${ }^{6}$ varia em torno de 0,05 a $0,07 \mathrm{mg} \mathrm{F} / \mathrm{kg}$. Para realizar este tipo de estimativa, é necessário considerar o preparo de alimentos e bebidas para crianças $^{22}$. Trabalhos mostram que mais de $50 \%$ do consumo diário de flúor é derivado da água ingerida ${ }^{31}$. Caso seja proveniente de água mineral embalada, às vezes a concentração de flúor é desconhecida devido à falta de informações precisas no rótulo, como é o caso de $10(9,6 \%)$ das águas avaliadas e comercializadas no Brasil, que contêm mais de 0,3 ppm F em sua composição (Tabelas 1, 2, 4, 5, 6). Por isso, em alguns casos o profissional opta pela suplementação, supondo que toda criança que mora numa cidade com fluoretação da água de abastecimento, mas que só consome água mineral, não está recebendo os benefícios deste método preventivo. Entretanto os resultados do presente estudo mostraram concentrações que variaram de 0,0 a 4,44 ppm $\mathrm{F}$, verificando-se que $18,3 \%$ das águas engarrafadas apresentaram mais de $0,3 \mathrm{ppm}$, sendo que $10,6 \%$ delas apresentaram mais de $0,7 \mathrm{ppm}$. Estes dados indicam, portanto, que é necessário o conhecimento da concentração de flúor da água mineral que o paciente infantil está consumindo, antes de optar pela suplementação, já que poderia estar consumindo água mineral com concentrações equivalentes às encontradas na água fluoretada de abastecimento público, fato que contra-indica sua prescrição. Poderia também estar ingerindo quantidades maiores, como é o caso de algumas águas encontradas nos Estados de São Paulo (S-1: 1,36 ppm F e S-2: 1,98 ppm F-), Santa Catarina (SC-1: 1,02 ppm F-), Paraná (PR-1: 0,92 ppm F), Rio Grande do Sul ( RG-4: 0,87 ppm F-, RG-2: 1,69 ppm F-, RG-3: 1,66 ppm F- e RG-1: 4,44 ppm F-) e também em duas águas de origem estrangeira provenientes da $\mathrm{Ar}$ gentina e Portugal, adquiridas no comércio da cidade de São Paulo, que apresentaram concentrações de 1,89 e 3,16 ppm F- respectivamente, as quais dependendo do seu consumo diário estariam submetendo o paciente infantil a uma sobredosagem e a um provável risco de fluorose dental. Sabe-se que para a instalação deste efeito colateral indesejado, é necessário um consumo de flúor superior a dose de segurança ${ }^{6}$ durante a fase de desenvolvimento dentário, capaz de alterar a mineralização dental ${ }^{20}$. O período denominado crítico de susceptibilidade para alterações estéticas que afetariam os incisivos centrais superiores (maxila), foi recentemente determinado como um intervalo de 4 meses que vai dos 22 aos 26 meses de idade ${ }^{12}$. A importância desses parâmetros torna-se portanto fundamental para os odontopediatras, pediatras e/ou clínicos gerais que mantêm um convívio profissional com crianças de baixa idade e que têm como objetivos bem definidos a manutenção da saúde geral.

Embora somente nas amostras de águas minerais dos Estados de SãoPaulo, Paraná, Santa Catarina e Rio Grande do Sul tenham sido encontradas altas concentrações de flúor, é possível que ocorram também em outros Estados do Brasil. Não só nesses Estados existem reservas de fluorita ${ }^{4}$, assim como em outras regiões litorâneas e do interior do País ${ }^{7,11}$, apresentando inclusive rios com fluoretação natural ${ }^{28}$. 
Baseado nos resultados do presente estudo, consideramos que um maior controle de qualidade das águas minerais industrializadas no Brasil faz-se necessário, com o propósito de prevenir a eventual ocorrência de fluorose dental em crianças, decorrentes da ingestão prolongada de águas com altos teores de flúor. Assim, considerando os presentes resultados e os conceitos atuais sobre a dose máxima de flúor que deve ser ingerida diariamente $(0,05$ a $0,07 \mathrm{mgF} / \mathrm{kg})$, podemos exemplificar que uma criança com $10 \mathrm{~kg}$ deveria ingerir no máximo 0,5 a $0,7 \mathrm{mg}$ F/ dia. Caso esteja então sendo consumida uma água mineral com 4,44ppm, só seriam necessários $158 \mathrm{ml}$ para atingir esta dose, o que representa aproximadamente, por exemplo, pouco menos de um copo da água RG-1 por dia, com esse teor. Este fato mostra que águas com altos teores de flúor estariam contra-indicadas para crianças menores de 7 anos de idade, o que deveria constar no rótulo do produto. Outro dado importante constatado ao analisar os rótulos das águas minerais, foi que não existe padronização na forma de apresentação do conteúdo de flúor. Alguns fabricantes apresentam a concentração como fluoreto de sódio $(\mathrm{NaF})$, fluoreto de potássio (KF), fluoretos ou $\mathrm{F}$. Seria aconselhável que o teor de flúor fosse apresentado em ppm F (flúor iónico ou fluoreto), com a finalidade de facilitar seu reconhecimento pois, apre-

\section{REFERÊNCIAS BIBLIOGRÁFICAS}

1. BOIVIN, G.; CHAVASSIEUX, P.; CHAPUY, M.C.; BAUD, C.A.; MEUNIER, P.J. Profil histomorfophométrique de la fluorose osseuse induite par l'ingestion prolongée d'eau de Vichy Saint-Yorre. Pathol. Biol., 34: 33-9, 1986.

2. BRASIL. Leis e Decretos. Código de águas minerais: Decreto-Lei n. 7.841 de 08 de ago. 1945. Diário Oficial da União, 20 ago. 1945. p. 194.

3. BRASIL. Ministério de Minas e Energia. Departamento Nacional de Produção Mineral. Sumário mineral. Brasília, 1994. v. 14

4. BRASIL. Ministério de Minas e Energia. Secretaria Nacional de Minas e Metalurgia. Departamento Nacional da Produção Mineral. Anuário mineral brasileiro. Brasília, 1991. v. 20.

5. BRASIL. Ministério da Saúde. Portaria n 56 / Dsb - 14 mar. 1977. Diário Oficial da União, 15 jun. 1977. Seção I - parte I.

6. BURT, B.A. The changing patterns of systemic fluoride intake. J. Dent. Res., 71: 1228-37, 1992. sentado na forma de compostos fluoretados seriam necessários cálculos para se estabelecer a dose em termos de risco e benefício.

Um maior controle de qualidade também evitaria que o consumidor, baseado no apelo promocional de algumas marcas comerciais, que colocam no rótulo chamadas como: "água mineral fluoretada", adquira um produto que fornece uma "exposição não terapêutica aos fluoretos" ${ }^{\prime 18}$.

As normas brasileiras que classificam as águas minerais como fluoretadas deveriam ser revistas, levando em consideração o binômio risco/benefício. Também, existe a necessidade de um sistema de vigilância sanitária nos níveis Federal, Estadual, ou Municipal, que regulamente a existência da presença de flúor natural em águas minerais.

\section{AGRADECIMENTOS}

À técnica do laboratório de Bioquímica Oral da FOP-UNICAMP, Mariza de Jesus Carlos Soares pela colaboração nas análises de flúor.

À química, Nadya de L. do Amaral do Serviço de Águas Subterrâneas do Departamento Nacional de Produção Mineral-Brasília, pelas informações fornecidas.
7. CAPELLA, L.F.; CARCERERI, D.L.; PAIVA, S.M.; ROSSO, R.A.; PAIXÃO, R.F.; SALTORI, E.K.; FREITAS, A.R.R.; ZENKNER, J.E.A.; BARROS FILHO, M.A. Ocorrência de fluorose dental endêmica. Rev. Goian. Odont., 37: 371-5, 1989.

8. CHAN, J.T.; STARK, C.; JESKE, A.H. Fluoride content of bottled waters: implications for dietary fluoride supplementation. Tex. Dent. J., 107: 17-21, 1990.

9. CLOVIS, J. \& HARGREAVES, J.A. Fluoride intake from beverage consumption. Community Dent. Oral Epidemiol., 16: 11-5, 1988.

10. DILLENBERG, J.S.; LEVY, S.M.; SCHOROEDER, D.C.; GERSTON, E.N.; ANDERSEN, C.J.C. Arizona providers'use and knowledge of supplements. Clin. Prev. Dent., 14: 15-26, 1992.

11. ELLWOOD, R.P.; CÔRTES, D.F.; O'MULLANE, D.M Fluoride exposure and enamel defects in Brazil. J. Dent. Res., 73: 331, 1994. 
12. EVANS, R.W. \& STAMM, J.W. An epidemiologic estimate of the critical period during which human maxillary central incisors are most susceptible to fluorosis. J. Public Health Dent., 51: 251-9, 1991.

13. FLAITZ, C.M.; HILL, E.M.; HICKS, M.J. A survey of bottled water usage by pediatric dental patients : implications for dental health. Quintessence Int., 20: 847-52, 1989.

14. FRANCO, F.C. \& MALTZ, M. A concentração de fluoretos em águas minerais, chás brasileiros e chimarrão. In: Reunião da Sociedade Brasileira de Pesquisas Odontológicas, 8. Águas de São Pedro, 1991. Anais. p. 4.

15. JASMIN, J.R.; IONESCO-BENAICHE, N.; MULLER, M. Latent fluorides: report of case. J. Dent. Child., 62: 220-3, 1995.

16. JONES, K.F. \& BERG, J.H. Fluoride supplementation: a survey of pediatricians and pediatric dentists. Am. J. Dis. Child., 146: 1449-91, 1992.

17. KUTHY, R.A. \& Mc TIGUE, D.J. Fluoride prescription practices of Ohio physicians. J. Public. Health Dent., 47: 172-6, 1987.

18. LALUMANDIER, J.A. \& ROZIER, R.G. The prevalence and risk factors of fluorosis among patient in a pediatric dental practice. Pediatr. Dent., 17: 19-24, 1995.

19. LANTZ, O.; JOUVIN, M.H.; DE VERNEJOUL, M.C.; DRUET, P. Fluoride induced chronic renal failure. Am. J. Kidney Dis., 10: 136-9, 1987.

20. LARSEN, M.J.; RICHARDS, A.; FEJERSKOV, O. Development of dental fluorosis according to age at start of fluoride administration. Caries Res., 19: 519-27, 1985.

21. LEVY, S.M. Systemic fluoride supplementation in an academic family practice setting. J. Fam. Pract., 24: 532-6, 1987.

22. LEVY, S.M.; KIRISTY, M.C.; WARREN, J.J. Sources of fluoride intake in children. J. Public. Health Dent., 55: 3952, 1995.

23. LEVY, S.M.; MAURICE, T.J.; JAKOBSEN, J.A. Feeding patterns, water sources and fluoride exposures of infants and 1-year-olds. J. Am. Dent. Assoc., 124: 65-9, 1993.

24. Mac FAYDEN, E.E.; McNEE, S.G.; WEETMAN, D.A. Fluoride content of some bottled spring waters. Br. Dent. J., 153: 423-4, 1982.

25. MARGOLIS, F.J.; BURT, B.A.; SCHORK, M.A.; BASHSHUR, H.L.; WHITTAKER, B.A,; BURNS, T.L. Fluoride supplements for children. Am. J. Dis. Child., 134: 865-8, 1980.
26. McGUIRE, S. Fluoride content of bottled water. N. Engl. J. Med., 321: 836-7, 1989.

27. MEUNIER, P.J.; FEMENIAS, M.; DUBOEUF, F.; CHAPUY, M.C.; DELMAS, P.D. Increased vertebral bone density in heavy drinkers of mineral water rich in fluoride. Lancet., $\mathbf{1}$ : $152,1989$.

28. SAMPAIO, F.C. \& BEZERRA, R. Dental fluorosis in Paraiba- Brazil. An epidemiological survey in geomedical interactins. In: World Congress on Preventive Dentistry, 5. São Paulo, 1995. Abstracts of papers. São Paulo, 1995. p. 46.

29. SCHALKA, M.M.S. \& RODRIGUES, C.R.M.D. A importância do médico pediatra na promoção da saúde bucal. Rev. Saúde Pública, 30: 179-86, 1996.

30. SOVIERO, V.M.; MASSAO, J.M.; RAMOS, M.E.; TURA, L.F. Posicionamento dos médicos pediatras frente a questões de odontopediatria. In: Reunião da Sociedade Brasileira de Pesquisas Odontológicas, 12. Águas de São Pedro, 1995. Anais, 1995. p. 123.

31. STANNARD, J.; ROVERO, J.; TSAMTSOURIS, A.; GAVRIS, V. Fluoride content of some bottled waters and recommendations for fluoride supplementation. J. Pedod., 14: 103-7, 1990.

32. TATE, W.H.; SNYDER, R.; MONTGOMERY, E.H.; CHAN, J.T. Impact of source of drinking water on fluoride supplementation. J. Pediatr., 117: 419-21, 1990.

33. TOUMBA, K.J.; LEVY, S.; CURZON, M.E.J. The fluoride content of bottled drinking waters. Br. Dent. J., 176: 266-8, 1994.

34. US ENVIRONMENTAL PROTECTION AGENCY. 1986. apud Newbrun, E. Current regulations and recommendations concerning water fluoridation, fluoride supplements, and topical fluoride agents. J. Dent. Res., 71: 1255-65, 1992.

35. VAN WINKLE, S.; LEVY, S.M.; KIRITSY, M.C.; HEILMAN, J.R.; WEFEL, J.S.; MARSHALL, T. Water and formula fluoride concentrations: significance for infants fed formula. Pediatr. Dent., 17: 305-10, 1995.

36. WEINBERGER, S.J. Bottled drinking waters: are the fluoride concentrations shown on the labels accurate? Int. J. Paed. Dent., 1: 143-6, 1991.

37. WORLD HEALTH ORGANIZATION. 1984. apud Newbrun, E. Current regulations and recommendations concerning water fluoridation, fluoride supplements, and topical fluoride agents. J. Dent. Res., 71: 1255-65, 1992. 